\title{
ARTICLE
}

Received 3 Mar 2016 | Accepted 7 Oct 2016 | Published 23 Nov 2016

DOI: $10.1038 /$ ncomms13539

OPEN

\section{Unlocking sperm chromatin at fertilization requires a dedicated egg thioredoxin in Drosophila}

Samantha Tirmarche ${ }^{1}$, Shuhei Kimura ${ }^{1}$, Raphaëlle Dubruille${ }^{1}$, Béatrice Horard ${ }^{1} \&$ Benjamin Loppin ${ }^{1}$

In most animals, the extreme compaction of sperm DNA is achieved after the massive replacement of histones with sperm nuclear basic proteins (SNBPs), such as protamines. In some species, the ultracompact sperm chromatin is stabilized by a network of disulfide bonds connecting cysteine residues present in SNBPs. Studies in mammals have established that the reduction of these disulfide crosslinks at fertilization is required for sperm nuclear decondensation and the formation of the male pronucleus. Here, we show that the Drosophila maternal thioredoxin Deadhead (DHD) is specifically required to unlock sperm chromatin at fertilization. In dhd mutant eggs, the sperm nucleus fails to decondense and the replacement of SNBPs with maternally-provided histones is severely delayed, thus preventing the participation of paternal chromosomes in embryo development. We demonstrate that DHD localizes to the sperm nucleus to reduce its disulfide targets and is then rapidly degraded after fertilization.

\footnotetext{
${ }^{1}$ Laboratoire de Biométrie et Biologie Évolutive, Université de Lyon, Université Lyon 1, CNRS, UMR 5558, Villeurbanne F-69622, France. Correspondence and requests for materials should be addressed to B.L. (email: benjamin.loppin@univ-lyon1.fr).
} 
$\mathrm{n}$ sexually reproducing animals, the differentiation of haploid spermatids into mature spermatozoa involves major reorganization of the nuclear architecture ${ }^{1}$. Starting as round nuclei after the second male meiotic division, spermatid nuclei slowly transform to eventually acquire the final, species-specific shape of mature sperm nuclei. Extreme compaction of nuclear DNA generally accompanies the streamlining of spermatids, resulting in the shutdown of basic nuclear activities, including transcription and DNA repair ${ }^{2}$. In most species, sperm nuclear compaction requires the massive replacement of somatic-type histones with sperm nuclear basic proteins (SNBPs) ${ }^{3,4}$. SNBPs encompass a heterogeneous group of chromosomal proteins that are specifically expressed in male germ cells and deposited during spermiogenesis 3,5 . Protamines, the best characterized SNBPs, are small (50-60 aa), arginine rich proteins present in vertebrates and some invertebrates ${ }^{6,7}$. A model based on mammalian protamines has proposed that these positively charged proteins bind the major groove of the double helix and form toroid-like structures containing about 50 kilobases of sperm $\mathrm{DNA}^{7-9}$. Protamines in eutherian mammals and a few other animal groups are also enriched in cysteine residues, which are otherwise rare in chromosomal proteins, including histones and most $\mathrm{SNBPs}^{3}$. During sperm maturation in eutherian mammals, oxidation of the protamine cysteine thiols $(-\mathrm{SH})$ allows the formation of a tridimensional network of disulfide bridges (-S-S-). Intermolecular disulfide crosslinks notably participate in the stabilization of sperm chromatin by connecting adjacent chromatin fibres $7,8,10,11$. It is actually well established that for most species of mammals, a thiol reducing agent, such as dithiothreitol (DTT), is required to elicit sperm nuclear decondensation in vitro. In addition, it has been alternatively proposed that in human sperm, protamines thiols are non-covalently bridged by Zinc $\left(\mathrm{Zn}^{2+}\right)$, thereby preventing or limiting the formation of excess disulfide bonds that could perturb sperm nuclear decondensation at fertilization ${ }^{12}$.

In this work, we functionally characterized the Drosophila maternal thioredoxin Deadhead (DHD) and we demonstrate that DHD is required to unlock sperm chromatin at fertilization. Thioredoxins are small redox proteins found in all organisms. They typically reduce disulfide bonds on target proteins using a pair of cysteine thiols present in their conserved active CGPC site ${ }^{13}$. Thioredoxins play important metabolic, protective or signalling functions but the molecular bases of their target specificity or functional specialization remain poorly understood $^{14}$. Three classical thioredoxins are found in Drosophila melanogaster. Trx-2 is a non-essential ubiquitous protein that participates in the protection against oxidative stress $^{15,16}$, whereas TrxT and DHD are sex-specific thioredoxins encoded by a pair of adjacent genes ${ }^{17,18}$. While the role of the testis-specific TrxT is unknown, DHD is specifically expressed in the female germline and is essential for embryo development ${ }^{19,20}$. In this work, we demonstrate that DHD is essential for sperm nuclear decondensation at fertilization.

\section{Results}

deadhead mutant females produce gynohaploid embryos. The original characterization of the dhd maternal effect phenotype showed that the vast majority of eggs produced by homozygous mutant females (hereafter referred to as $d h d$ eggs) were fertilized but failed to develop ${ }^{19}$. Interestingly, Salz and colleagues also observed that about $5 \%$ of $d h d$ embryos reached late embryogenesis and showed severe head developmental defects ${ }^{19}$. We noticed that these defects were reminiscent of the incomplete head involution typically observed in haploid Drosophila embryos $^{21-23}$. In addition, early mention of sperm nuclear decondensation defects in dhd eggs (see ref. 24) prompted us to reinvestigate the $d h d$ phenotype in detail.

The original $d h d^{J 5}$ allele is a $1.4 \mathrm{~kb}$ deficiency on the $\mathrm{X}$ chromosome that disrupts $d h d$ and the immediately adjacent, paralogous gene $\operatorname{Tr} x-T$. As $\operatorname{Tr} x$ - $T$ is strictly expressed in the male germline ${ }^{18}$, the $d h d^{J 5}$ allele can be used to specifically address the maternal function of $d h d^{19}$. In addition, the $d h d$ maternal effect embryonic lethal phenotype is fully rescued by a genomic transgene containing dhd but not $\operatorname{Tr} x-T^{19}$ (Fig. 1a; Table 1). To test the possibility that late $d h d$ embryos could represent haploid escapers of the early arrest phenotype, we crossed $d h d^{J 5}$ mutant females with males homozygous for a cid-GFP transgene. In contrast to embryos from a control cross that zygotically expressed the centromeric marker CID::GFP in all cells, none of

a

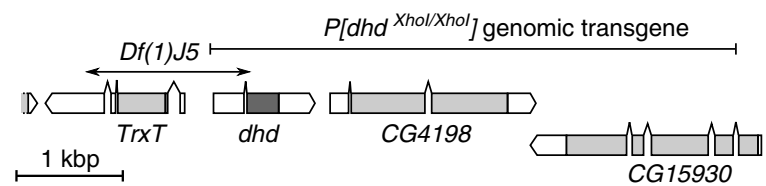

b
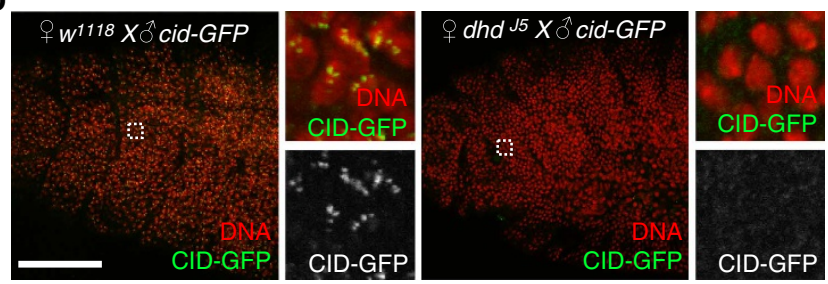

C
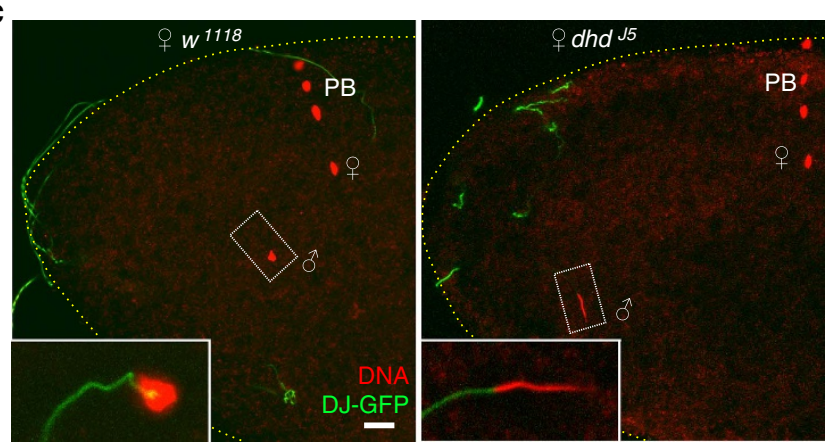

d

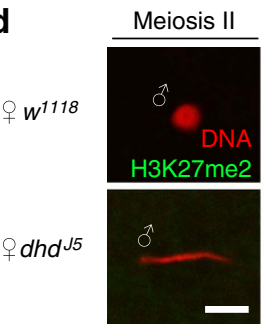

Cycle 1
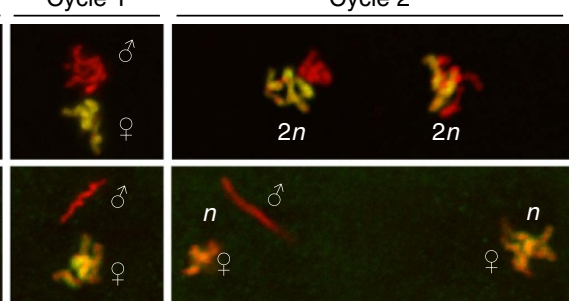

Figure 1 | DHD is required for sperm nuclear decondensation. (a) The dhd genomic region ( $4 \mathrm{~F} 4, \mathrm{X}$ chromosome) showing the genomic $P[$ dhd $X$ hol/Xhol] rescue transgene and the $D f(1) J 5$ deletion that disrupts dhd and TrxT. (b) Zygotic expression of the paternally-inherited cid-GFP transgene, which expresses a GFP-tagged centromeric histone (insets), is detected in late control embryos but not in dhd embryos. Scale bar, $50 \mu \mathrm{m}$. (c) At fertilization, the sperm nucleus (inset) fails to decondense in dhd eggs. The sperm flagellum is stained with the Don Juan(DJ)-GFP marker (green) DNA is in red. Note that the four female meiotic products are visible in both eggs. Scale bar, $10 \mu \mathrm{m}$. (d) The sperm nucleus does not participate in the early zygotic mitoses in dhd embryos. Maternal chromosomes are visualized with anti-H3K27me2 staining (green) and appear yellow. PB: polar bodies. Scale bar, $5 \mu \mathrm{m}$. 
Table 1 | Embryo hatching rates.

\section{Genotype of females}

$w^{1118}$

$w^{*}, D f(1) d h d^{15} / w^{*}, D f(1) d h d^{15}$

$w^{*}, D f(1) d h d^{15} / D f(1) J C 70$

$w^{\star}, D f(1) d h d^{15} / w^{\star}, D f(1) d h d^{15} ; C y O, P\left[d h d^{X h o l} / X^{*}\right.$ hol,$\left.w^{+}\right] /+$

$w^{*}, D f(1) d h d^{15} / w^{*}, D f(1) d h d^{15}$

$w^{*}, D f(1) d h d^{15 /} w^{*}, D f(1) d h d^{15} ; P\left[d h d^{W T}, w^{+}\right] / P\left[d h d^{W T}, w^{+}\right]$

$w^{*}, D f(1) d h d^{J 5} / w^{*}, D f(1) d h d^{15} ; P\left[d h d^{C 34 S}, w^{+}\right] / P\left[d h d^{C}{ }^{\prime 34 S}, w^{+}\right]$
Genotype of males

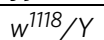

$w^{1118} / Y$

$W^{1118} / Y$

$w^{1118} / Y$

$w^{1118} / Y ; \Delta M s t 35 B / \Delta M s t 35 B$

$w^{1118} / Y$

$W^{1118} / Y$
No. of eggs

264

577

489

136

328

251

955
Hatch. rate (\%)

90.9

0

0

80.1

0

80.5

0.2

The $D f(1) d h d^{15}$ deficiency and the $P\left[d h{ }^{X}\right.$ Xhol/Xhol] genomic rescue transgene are described in Fig. 1a. $D f(1) J C 70$ is a large deficiency uncovering the dhd locus. $\Delta M s t 35 B$ mutation is described in ref. 39. $P\left[d h d^{W T}\right]$ and $P\left[d h d^{C 34 S}\right]$ transgenes are described in the text. $w^{\star}$ indicates an unspecified mutant allele of the white gene.

the dhd embryos that reached gastrulation expressed the paternal marker (Fig. 1b). Direct observations of mitotic figures in $d$ hd embryos were also indicative of haploid development (Supplementary Fig. 1). We conclude that rare dhd embryos escaping early arrest develop as gynohaploids after the loss of paternal chromosomes.

dhd affects sperm nuclear decondensation. We then examined fertilization and zygote formation in $d$ hd eggs to follow the fate of paternal chromosomes. As for other insects, fertilization is internal in Drosophila and the needle-shaped, extremely compact sperm nucleus immediately initiates decondensation after its delivery in the egg cytoplasm ${ }^{25}$. Accordingly, during the second female meiotic division (the earliest stage that can be practically observed), the sperm nucleus in wild-type eggs typically appears roundish or at least partially decondensed. In striking contrast, the sperm nucleus systematically retained its original needleshape in fertilized dhd eggs, showing no sign of decondensation (100\%, $n=66$; Fig. 1c). Observation of early syncytial divisions in dhd embryos confirmed that the paternal genome, still compacted in the sperm nucleus, does not participate in development, thus providing an explanation for the origin of gynohaploid embryos (Fig. 1d).

The female pronucleus often fails to migrate in $d$ hd eggs. In Drosophila, female meiosis resumes at egg activation, which occurs shortly after ovulation and independently of fertilization. With its two spindles organized in tandem, the second meiotic division generates four meiotic products typically aligned orthogonally to the antero-dorsal egg surface. In fertilized eggs, the innermost meiotic product then migrates towards the male nucleus and becomes the female pronucleus. Pronuclear migration occurs along microtubules of the giant sperm aster, which is nucleated from the paternally-inherited centrioles ${ }^{25,26}$. In contrast to the conclusion of the original report ${ }^{19}$, we observed that female meiosis resumed normally $(100 \%, n=17)$ in $d h d$ eggs, with the four meiotic products aligned in a way indistinguishable to wild-type eggs (Fig. 1c). We however noticed that, subsequently, the female pronucleus frequently (78\%, $n=586)$ failed to migrate toward the sperm nucleus and instead remained at the egg periphery with the three other products, as in wild-type unfertilized eggs (Fig. 2a). Pronuclear migration also fails in eggs fertilized by sperm from paternal effect male sterile mutants affecting sperm plasma membrane (SPM) breakdown, such as sneaky (snky), misfire $(m f r)$ and wasted $(w s t)^{27-29}$. SPM breakdown is indeed not only critical for sperm nuclear decondensation but is also required for the release of paternal centrioles in the egg cytoplasm and the formation of the sperm aster. To determine if persistence of the plasma membrane on the sperm nucleus could account for the dhd phenotype, we crossed control and dhd mutant females with males expressing Snky-GFP ${ }^{30}$. Snky-GFP is a specific marker of the sperm acrosome, a membrane-bound vesicular structure at the anterior tip of the sperm nucleus. After fertilization in Drosophila, the acrosome normally detaches from the sperm nucleus after the removal of SPM and remains at close proximity in the egg cytoplasm ${ }^{30}$. Interestingly, in dhd eggs, the acrosome remained connected to the anterior extremity of the sperm nucleus, opening the possibility that SPM breakdown was not completed in $d$ hd eggs (Fig. $2 \mathrm{~b}$ ). Anti- $\alpha$-tubulin immunostaining however revealed that a sperm aster was systematically present in dhd eggs, in sharp contrast to eggs fertilized with snky mutant sperm where this structure is never observed (ref. 27 and Supplementary Fig. 2). Although this result demonstrates that SPM breakdown is at least initiated in $d h d$ eggs, we noted that the sperm aster appeared abnormally small compared with wild-type eggs observed at the same stage (Fig. 2c). Additionally, we observed that the zygotic centrosome was poorly defined in $d h d$ eggs and remained associated to the needle-shape nucleus (Fig. 2d). In early zygotes, however, the centrosome duplicated and eventually detached from the sperm nucleus (Fig. 2d). These observations suggest that the release of paternal centrioles is delayed in $d$ d d eggs, which in turn likely affects the recruitment of pericentriolar material and the timely growth of the sperm aster.

Replacement of SNBPs with histones is delayed in $d$ hd eggs. Drosophila melanogaster sperm nuclei almost entirely lack histones and sperm DNA is instead packaged with at least four SNBPs: the almost identical Mst35Ba and Mst35Bb paralogous proteins (also known as ProtA and ProtB, respectively), Mst77F and the recently identified Prtl99 $\mathrm{C}^{31,32}$. In contrast to protamines, Drosophila SNBPs are substantially larger proteins (144-215 aa) that contain equivalent levels of arginine and lysine basic residues. In addition, these protamine-like proteins all possess a motif related to the High Mobility Group (HMG) box found in insects but not in mammals ${ }^{32,33}$. At fertilization, Drosophila SNBPs are rapidly removed from the sperm nucleus and immediately replaced with maternally-provided histones in a process that requires the nucleosome assembly complex HIRA $^{34-36}$. To analyse the replacement of SNBPs with histones in dhd eggs, we crossed mutant females with males expressing GFPtagged versions of the fly SNBPs (refs 31,37). Strikingly, we observed that the sperm nucleus systematically retained SNBPs in dhd eggs (Fig. 2a) and failed to incorporate histones (100\%, $n=33$ ) (Fig. 3a,b). Importantly, we observed an identical and equally penetrant phenotype in eggs from $d h d^{5} / D f(1) C 70$ females, where $D f(1) C 70$ is a chromosomal deletion that encompasses the dhd genomic region (Supplementary Fig. 3; Table 1). Furthermore, this phenotype was fully rescued by a transgene containing the genomic $d h d$ sequence (Fig. $3 \mathrm{~b}$ and Table 1), thus demonstrating that the observed phenotype is caused by the loss of $d h d$ function. 
a

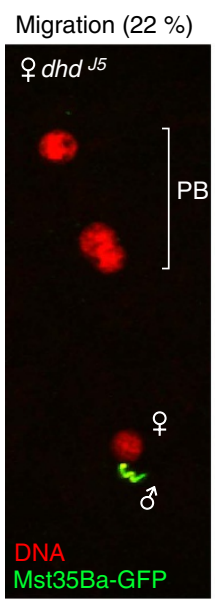

d

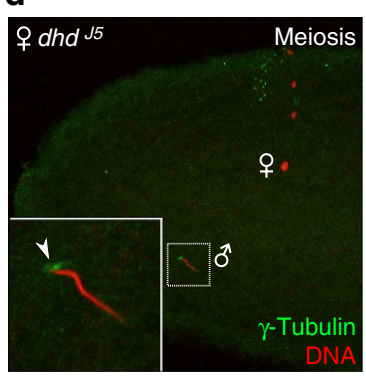

b
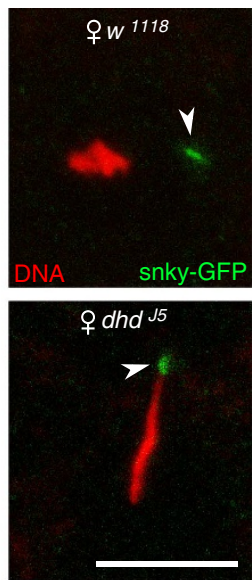

No migration $(78 \%)$

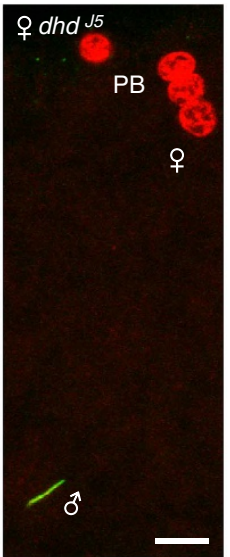

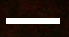

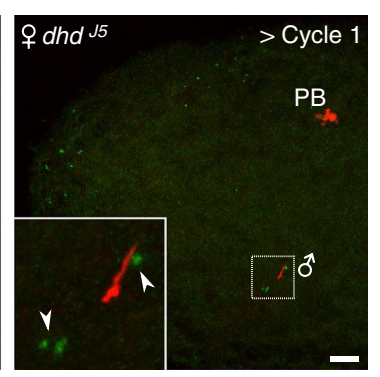

c
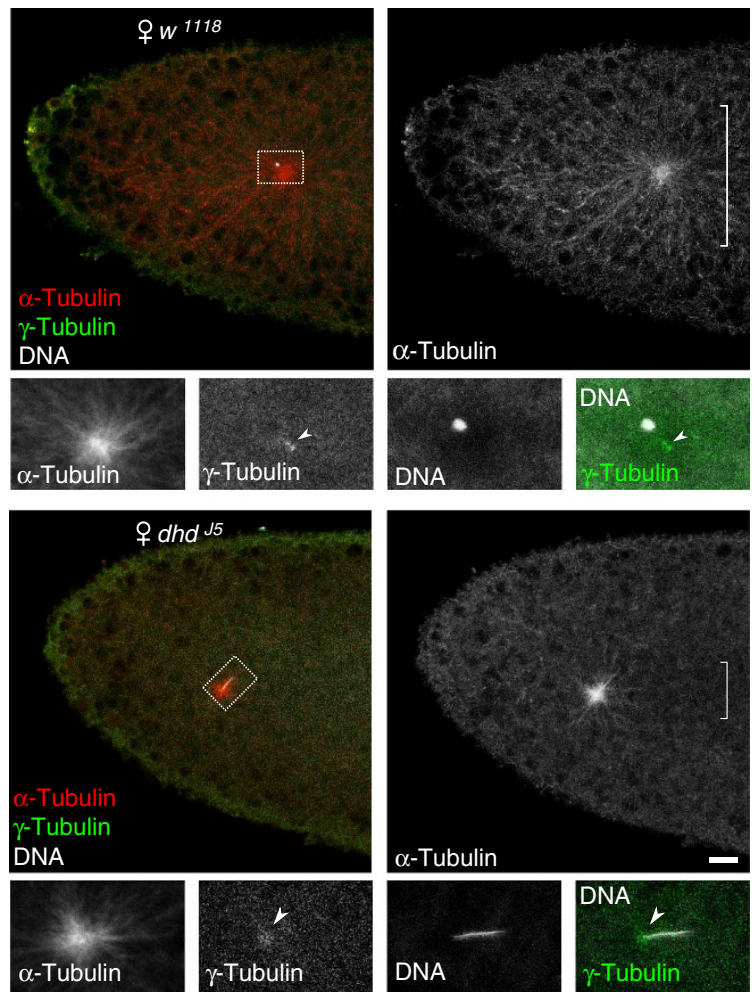

Figure 2 | Sperm aster formation and pronuclear migration are affected in dhd eggs. (a) Left: a dhd egg at pronuclear apposition. The three polar bodies (PB) are visible. Right: a dhd egg in which the female pronucleus failed to migrate and remained associated with the polar bodies. The respective percentage of each phenotype is indicated $(n=586)$. Sperm nuclei were visualized using the paternal Mst35Ba-GFP transgene (green). DNA is stained with propidium iodide (red). (b) Top panel: a $w^{1118}$ egg during meiosis II showing the male nucleus and the sperm acrosome (arrowhead). Bottom: a dhd eggs during meiosis II with the acrosome still associated to the anterior extremity of the sperm nucleus (arrowhead). The acrosome is visualized with the paternal Snky-GFP marker. (c) Eggs in telophase of meiosis II from $w^{1718}$ and $d h d^{J 5}$ females stained for DNA (white), $\alpha$-tubulin (red) and $\gamma$-tubulin (green) to reveal nuclei, the sperm aster and the centrosomes, respectively (the female meiotic products are not visible on these confocal sections). In $w^{1718}$ eggs (upper panels), the anti- $\gamma$-tubulin stains the zygotic centrosome (which contains a pair of sperm centrioles) at proximity of the male pronucleus (100\%, $n=25$; arrowhead, inset). In dhd eggs (lower panels), the centrosome appears diffused and almost systematically associated (98\%, $n=53)$ to the compacted sperm nucleus (arrowhead, inset). The brackets indicate the extent of the sperm aster in $w^{1118}$ and dhd ${ }^{15}$ eggs. (d) Left: a dhd egg in telophase of meiosis II. Right: a dhd embryo after the end of cycle 1. The centrosomes (arrowheads, inset) have duplicated and detached from the male nucleus. The rosette of polar body (PB) chromosomes is visible. Green: $\gamma$-tubulin, red: DNA. Scale bars, $10 \mu \mathrm{m}$.

Interestingly, in dhd embryos that had reached or completed the first zygotic cycle, histones were eventually detected in the sperm nucleus despite the persistence of SNBPs (Fig. 3b and Supplementary Fig. 3). To confirm that this histone staining reflected de novo assembly of nucleosomes on paternal chromatin, we performed anti-histone immunostainings on embryos produced by dhd Hira double mutant females. We used the Hira $^{\text {ssm }}$ point substitution allele (R225K), which does not affect female viability or fecundity but strongly impacts de novo nucleosome assembly in the male pronucleus ${ }^{34}$. In $d$ dh embryos observed during nuclear cycle 1 or beyond, histones were almost systematically detected in the needle-shaped sperm nucleus $(95.3 \%, n=65)$. In clear contrast, the sperm nucleus did not contain detectable levels of histones in a majority of dhd Hira double mutant embryos observed during the same developmental time window $(74.2 \%, n=31)$, the rest of embryos showing only a very weak staining in the sperm nucleus that we attribute to residual Hira activity ${ }^{34}$ (Supplementary Fig. 4). These results confirmed that SNBP/histone replacement is dramatically delayed in $d h d$ eggs, thus preventing male pronuclear formation.

To more directly test the possibility that the dhd nuclear phenotype was indeed caused by a delay in SNBP removal, we took advantage of the fact that deletion alleles of both $M s t 35 \mathrm{Ba} / \mathrm{b}$ protamine-like genes do not prevent the production of fertilization-competent sperm $^{38,39}$. We reasoned that the previously reported impact of $M s t 35 \mathrm{Ba} / \mathrm{b}$ deletion on sperm chromatin compaction ${ }^{32,38}$ could facilitate the SNBP/histone replacement at fertilization in dhd mutant eggs. Crossing dhd mutant females with males homozygous for a knock-out of both Mst35B copies ( $4 M s t 35 B$ ) (ref. 39) did not restore embryo viability (Table 1). However, we observed a partial but clear rescue of sperm chromatin remodelling dynamics, as histone deposition was detected before the end of female meiosis in this context (Fig. 3c). In a majority of cycle 1 embryos, sperm nuclei appeared partially decondensed and the SNBP marker Mst77F-GFP was replaced with histones. Finally, in later embryos, the male nucleus frequently $(76 \%, n=81)$ appeared as a mass of chromosome-like structures, a phenotype relatively rare $(7 \%, n=91)$ in control dhd embryos collected for the same period of time (Fig. 3c). We conclude that DHD is critically required for the rapid removal of SNBPs from the sperm nucleus at fertilization.

Drosophila sperm chromatin is locked by disulfide bonds. Previous work demonstrated that the typical WCGPCK redox catalytic motif of DHD was essential for its function ${ }^{20}$. We thus 
a

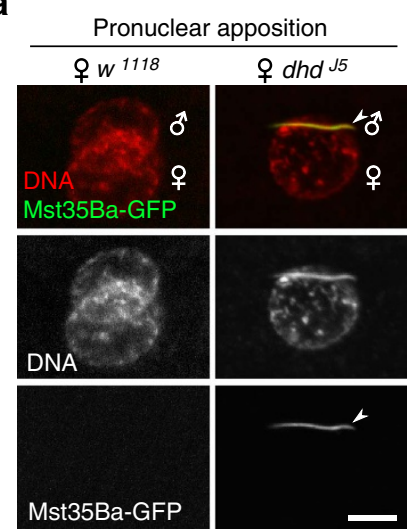

b

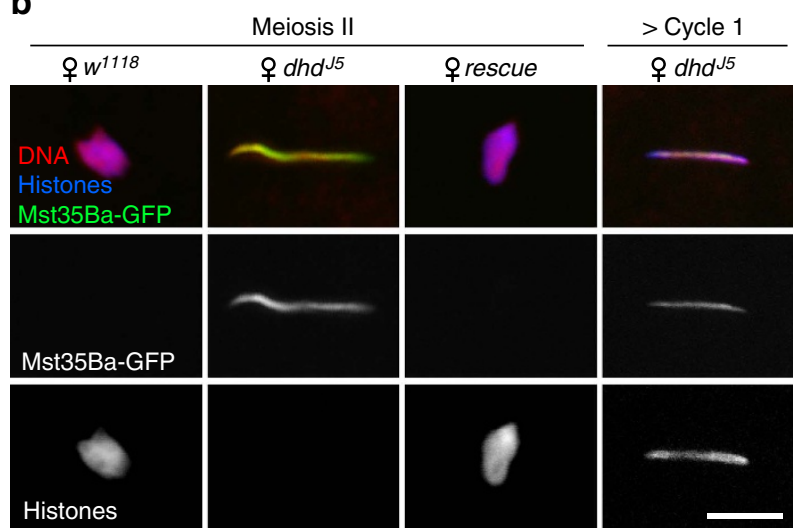

C

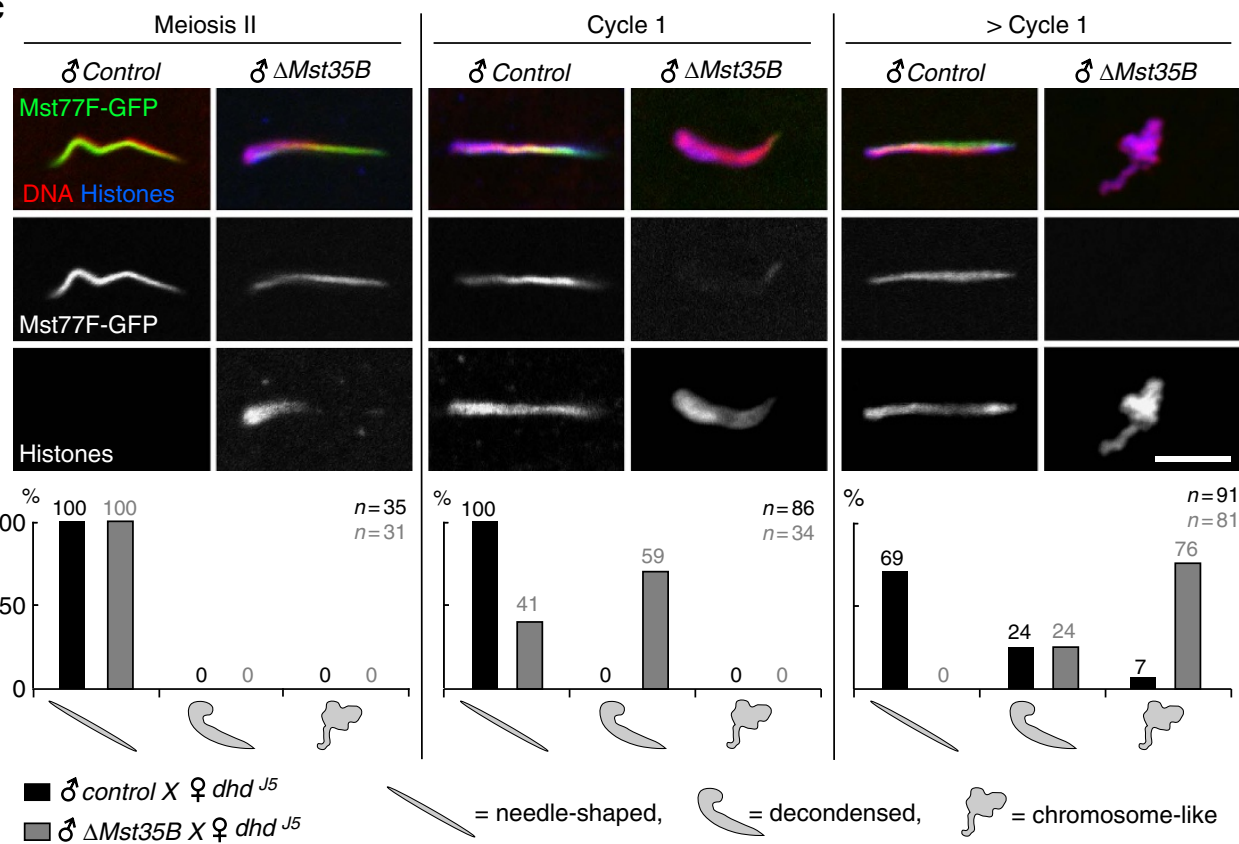

Figure 3 | DHD is required for the timely removal of SNBPs at fertilization. (a) Pronuclear apposition in eggs from control ( $w^{1118}$ ) or dhd ${ }^{15}$ females mated with transgenic Mst35Ba-GFP males. The sperm nucleus (arrowhead) in dhd eggs still contains Mst35Ba-GFP (green). (b) Eggs or cycle 1 embryos from $w^{1118}$, $d h d^{15}$ or $d h d^{15} ; P\left[d h d^{X h o l / X h o l}\right]$ (rescue) females mated with Mst35Ba-GFP males. The replacement of SNBPs with histones is severely delayed in dhd eggs and the phenotype is fully rescued by the genomic transgene. (c) Dynamics of SNBP/histone replacement in dhd eggs fertilized by control (Mst77F-GFP) or mutant ( $\triangle$ Mst35B; Mst77F-GFP) sperm. Distribution of phenotypic classes is shown for each stage. '> Cycle 1 ' indicates embryos with the polar body condensed into a rosette of chromosomes. Scale bars, $5 \mu \mathrm{m}$.

wondered whether DHD was involved in the reduction of putative disulfide bonds present on sperm chromatin. Remarkably, all four Drosophila SNBPs identified so far show conserved cysteine residues, thus opening the possibility that they could be involved in the formation of disulfide bonds in a way similar to mammalian protamines (refs 31,32,40,41; Supplementary Fig. 5). We thus tested the effect of the disulfide reducing agent DTT on the dynamics of sperm nuclear decondensation in vitro. Mature Mst35Ba-GFP spermatozoa obtained from dissected seminal vesicles remained basically inert in a non-reducing control buffer, showing no evidence of nuclear decompaction after $30 \mathrm{~min}$ of incubation. Strikingly, incubation of sperm nuclei in the presence of $2 \mathrm{mM}$ DTT induced dramatic nuclear decondensation and SNBP removal (Fig. 4). Nuclear swelling appeared maximal in the central region of the nuclei, which also showed the strongest reduction of Mst35BaGFP fluorescence. In contrast, both extremities of DTT-treated nuclei remained relatively compacted with a higher density of SNBP (Fig. 4d). Incubation with DTT also increased the amount of free thiol groups in sperm nuclei after $10 \mathrm{~min}$, as measured using the fluorescent marker monobromobimane $(\mathrm{mBrB})$ (Fig. 4c). After $30 \mathrm{~min}$ exposure to DTT, however, $\mathrm{mBrB}$ fluorescence decreased with the exception of nuclear extremities that still contained higher levels of SNBP. These observations suggest that after the $10 \mathrm{~min}$ treatment, $\mathrm{mBrB}$ is crosslinked to free thiol groups on SNBPs generated by the reduction of disulfide bonds. A 30 min DTT treatment was however required to induce significant sperm nuclear decompaction and SNBP removal. The eventual decrease of $\mathrm{mBrB}$ fluorescence observed after $30 \mathrm{~min}$ incubation presumably reflects the eviction of SNBPs. Taken together, our results strongly suggest that Drosophila sperm chromatin is stabilized with disulfide bridges, thus opening the possibility that DHD could be required for their reduction at fertilization.

DHD directly targets sperm chromatin. Any direct implication of DHD in the reduction of SNBP disulfide crosslinks would 
a
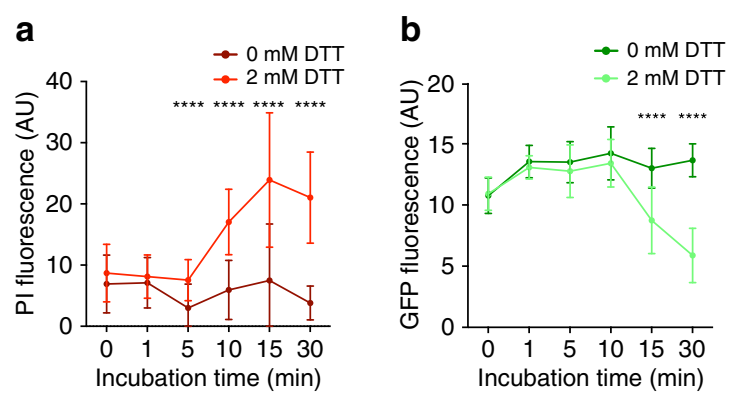

C

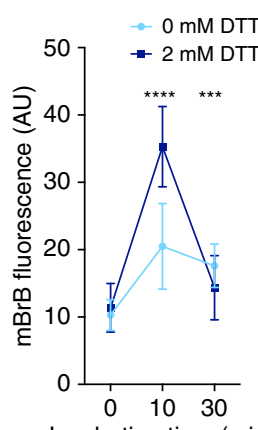

d

$2 \mathrm{mM}$ DTT - $0 \mathrm{~min}$
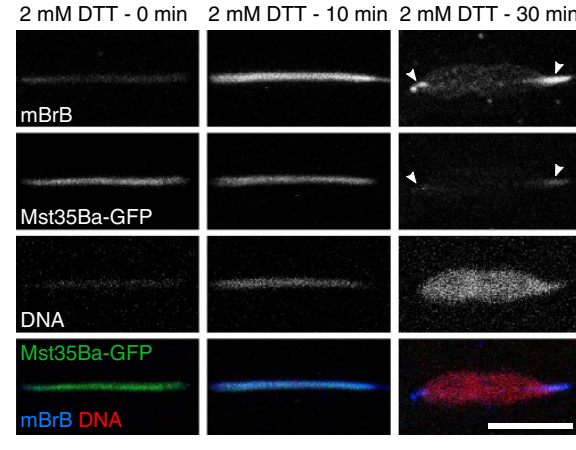

Figure 4 | DTT induces SNBP eviction and sperm nuclear decondensation in vitro. Mst35Ba-GFP sperm dissected from seminal vesicles were incubated with DTT for the indicated time and stained with propidium iodide (PI, red) and $\mathrm{mBrB}$ (blue). (a-c) Quantification of PI (a), native GFP (b) and $\mathrm{mBrB}$ (c) fluorescent signals in representative experiments (Mann-Whitney test. ${ }^{\star \star \star} P<0.001$. ${ }^{\star \star \star \star} P<0.0001$ ). Error bars indicate SD calculated for 29 analysed nuclei for each time point. (d) Confocal images of Mst35Ba-GFP sperm nuclei incubated with DTT for the indicated time. Arrowheads indicate condensed nuclear regions positive for both $\mathrm{mBrB}$ and Mst35Ba-GFP. Scale bar: $5 \mu \mathrm{m}$.

require its preloading in eggs and immediate targeting to the sperm nucleus at fertilization. We first studied the expression and distribution of DHD protein using a specific polyclonal antibody. Western-blotting analyses indeed confirmed that DHD was specifically expressed in ovaries and then maternally deposited in eggs (Fig. 5a,b; Supplementary Fig. 6). At fertilization, DHD appeared very abundant and homogeneously distributed throughout the egg cytoplasm. Surprisingly however, DHD became rapidly undetectable after completion of the first zygotic cycle, thus indicating that this thioredoxin likely plays no other role after zygote formation (Fig. 5c). The rapid removal of SNBPs from the fertilizing sperm nucleus, which is completed before egg deposition $^{25}$, precluded any direct observation of DHD during this process. In an attempt to circumvent this difficulty, we generated transgenic flies expressing a mutated version of $\mathrm{DHD}\left(\mathrm{DHD}^{\mathrm{C} 34 \mathrm{~S}}\right)$, in which the replacement of the second cysteine residue in the WCGPCK catalytic motif is predicted to trap the thioredoxin as a covalent intermediate species with its target proteins ${ }^{13}$ (Fig. 6a,b). In contrast to the fully functional $P\left[d h d^{W T}\right]$ control transgene, the $P\left[d h d^{C 34 S}\right]$ transgene did not rescue the fertility of dhd mutant females despite normal expression (Table 1; Supplementary Fig. 7). As expected, in dhd eggs rescued with the control transgene, the sperm nucleus appeared normally decondensed and DHD was only detected in the egg cytoplasm. Strikingly however, in about $30 \%$ of dhd; $P\left[d h d^{C 34 S}\right]$ eggs, we observed the specific accumulation of $\mathrm{DHD}^{\mathrm{C} 34 \mathrm{~S}}$ in the needle-shaped sperm nucleus (Fig. 6c; Supplementary Fig. 8). Importantly, DHD ${ }^{\mathrm{C} 34 \mathrm{~S}}$ was only detected on the sperm nucleus and not on the sperm tail or on the acrosomal region. In the rest of observed eggs, $\mathrm{DHD}^{\mathrm{C} 34 \mathrm{~S}}$ was detected in the cytoplasm but not in the sperm nucleus, again suggesting incomplete sperm plasma membrane breakdown in a fraction of mutant eggs. These results however strongly suggest that DHD directly reduces target disulfides present on sperm chromatin.

DHD induces sperm nuclear decondensation in vitro. To directly address the ability of DHD to perform sperm nuclear decondensation, we tested the activity of purified recombinant thioredoxins on isolated sperm nuclei in vitro. We cloned, bacterially-expressed and purified the wild-type and C34S mutant versions of DHD. As an additional control, we also purified Trx-2, the ubiquitously expressed Drosophila thioredoxin (Supplementary Fig. 9). The 6-histidine-tagged purified thioredoxins were reduced using DTT before their use in the assay. Incubation of Mst35Ba-GFP sperm nuclei with either Trx-2 or $\mathrm{DHD}^{\mathrm{C} 34 \mathrm{~S}}$ had no detectable effect on sperm nuclear compaction or the level of Mst35Ba-GFP fluorescence. In clear contrast, incubation of sperm nuclei with wild-type recombinant DHD protein induced both sperm nuclear decondensation and Mst35Ba-GFP removal in a way similar to the effect of $2 \mathrm{mM}$ DTT treatment (Fig. 7). These experiments strongly support a direct role of DHD in SNBP removal and sperm nuclear decondensation. Furthermore, the lack of detectable activity of Trx-2 in this assay suggests that DHD presents unique properties not shared with other thioredoxins.

\section{Discussion}

In this study, we have identified the Drosophila maternal thioredoxin Deadhead as critically required for sperm nuclear decondensation at fertilization. We propose that DHD is involved in the reduction of disulfide crosslinks that connect cysteine residues of SNBPs in mature sperm chromatin.

The stabilization of sperm chromatin with disulfide bonds is a remarkable innovation that emerged independently in distantlyrelated animal groups with the selective acquisition of cysteine residues in $\mathrm{SNBPs}^{3,42,43}$. In this work, we first established that Drosophila sperm chromatin, which is packaged with cysteinerich SNBPs, is stabilized by disulfide crosslinks. We have shown that the reducing agent DTT allows efficient decompaction of Drosophila sperm nuclei in vitro, in a way similar to its wellestablished activity on sperm nuclei from eutherian mammals, for instance $^{44}$. Similarly, it has been recently shown that pretreatment of Drosophila sperm nuclei with DTT improves antibody accessibility to nuclear epitopes in immunofluorescence experiments ${ }^{32}$. Although little is known about the structural organization of Drosophila sperm chromatin, a recent study showed that the Drosophila SNBP Mst77F is a DNA binding protein which induces DNA condensation in vitro through a multimerization process involving its coiled-coil domain ${ }^{45}$. Oxidation of cysteine thiols present in Mst77F could participate in the stabilization of this unique chromatin architecture through the establishment of disulfide crosslinks. Disulfide bonds could also allow the formation of inter-chromatin fibre associations, as proposed for mammalian sperm chromatin $^{8}$.

The implication of DHD in the reduction of sperm chromatin disulfide crosslinks in Drosophila eggs is first supported by our detailed characterization of the dhd phenotype. Among the rare Drosophila maternal effect mutants affecting sperm chromatin remodelling $33,34,46$, dhd is unique and shows the earliest phenotype reported so far. Notably, in dhd mutant eggs, the sperm nucleus is initially intact, showing no sign of decondensation. Second, any role of DHD in the reduction of 


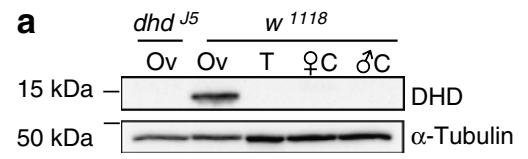

C

b
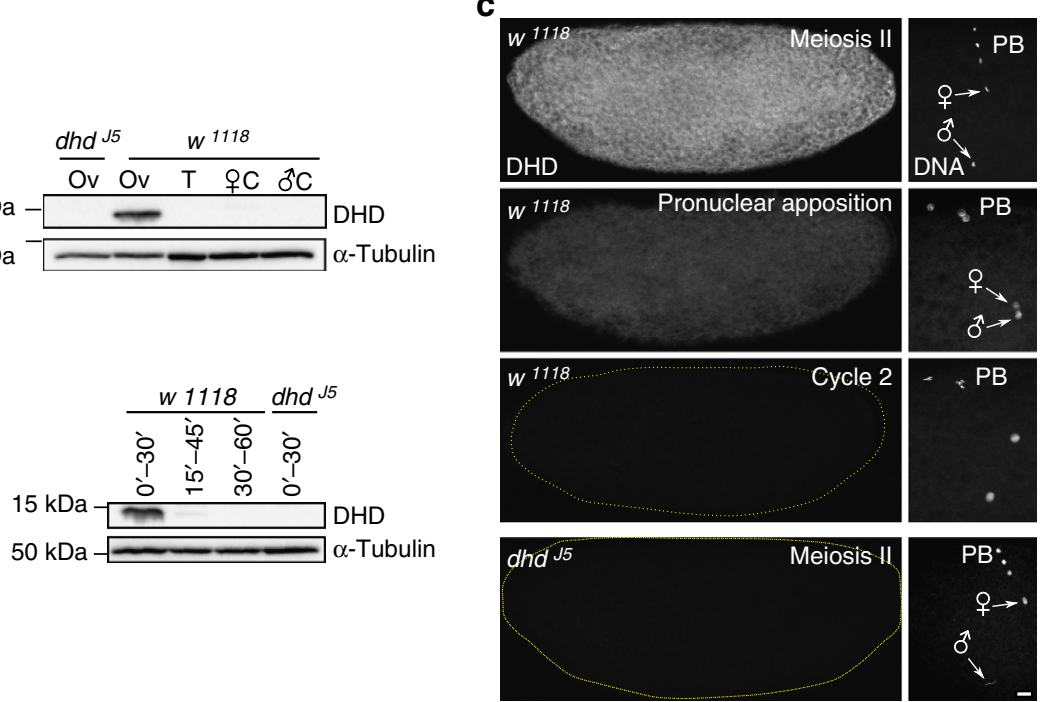

Figure 5 | Expression and distribution of maternally-expressed DHD protein. (a-b) Western blot analyses of DHD expression in adult tissues (a) or embryos collected during the indicated time windows after egg laying (b). $\boldsymbol{\alpha}$-tubulin was used as loading control. Ov: ovaries, T: testis, C: carcasses. See also Supplementary Fig. 6. (c) Confocal images of eggs or embryos laid by $w^{1118}$ or $d d^{15}$ females and stained for DHD (left) and DNA (right). A close-up of the region containing the nuclei is shown on the right (arrows: male and female nuclei; PB: polar bodies). Scale bar, $10 \mu \mathrm{m}$

a Reduction of target disulfide bond

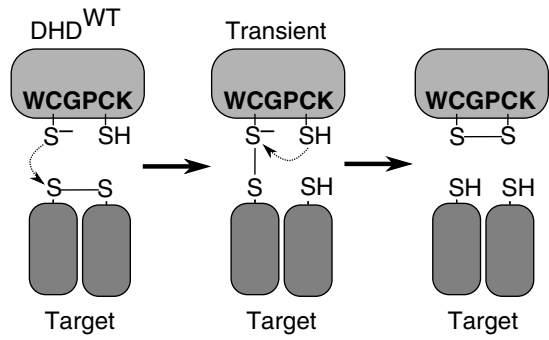

C
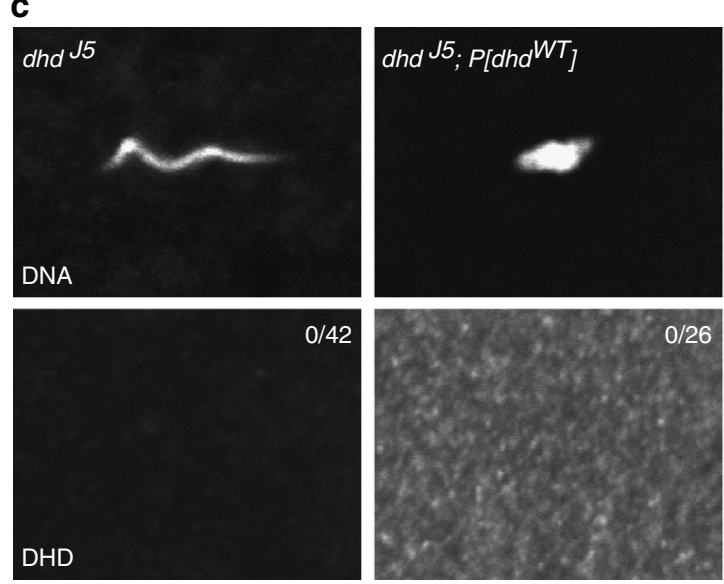

b Covalent trapping reaction
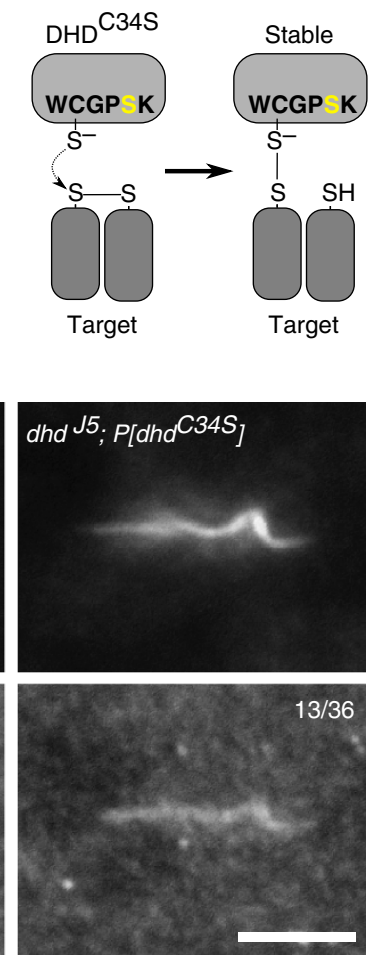

Figure 6 | DHD is specifically targeted to the sperm nucleus at fertilization. (a) Mechanism of disulfide bond reduction on target proteins by wild-type DHD. (b) Strategy for trapping DHD on its targets: the replacement of the resolving cysteine with a serine (C34S) in the WCGPCK motif is predicted to stabilize the mixed-disulfide between DHD and its target. (c) Sperm nuclei in eggs laid by females of the indicated genotypes stained for DNA and with the anti-DHD antibody. The number of DHD-positive sperm nuclei is indicated for each genotype. See also Supplementary Fig. 8. Scale bar, $5 \mu$ m.

sperm nuclear disulfide bonds implies the critical requirement of its catalytic redox motif. Salz and colleagues previously demonstrated that transgenes expressing DHD in which the two active cysteines are replaced by serines failed to rescue the fertility of mutant females ${ }^{20}$. In this work, we have shown that replacing the second cysteine residue of the redox motif not only abolished DHD function but also trapped the protein on sperm chromatin in a remarkably specific manner. This experiment strongly suggests that DHD directly targets disulfide bonds on sperm chromatin. Interestingly, the $\mathrm{DHD}^{\mathrm{C} 34 \mathrm{~S}}$ mutant protein could not rescue the dhd nuclear phenotype despite its expected ability to cleave its disulfide targets (Fig. 6b). We speculate that the irreversible covalent trapping of DHD ${ }^{\mathrm{C}} 4 \mathrm{~S}$ on SNBPs at the surface of sperm chromatin could impede their eviction and 
a
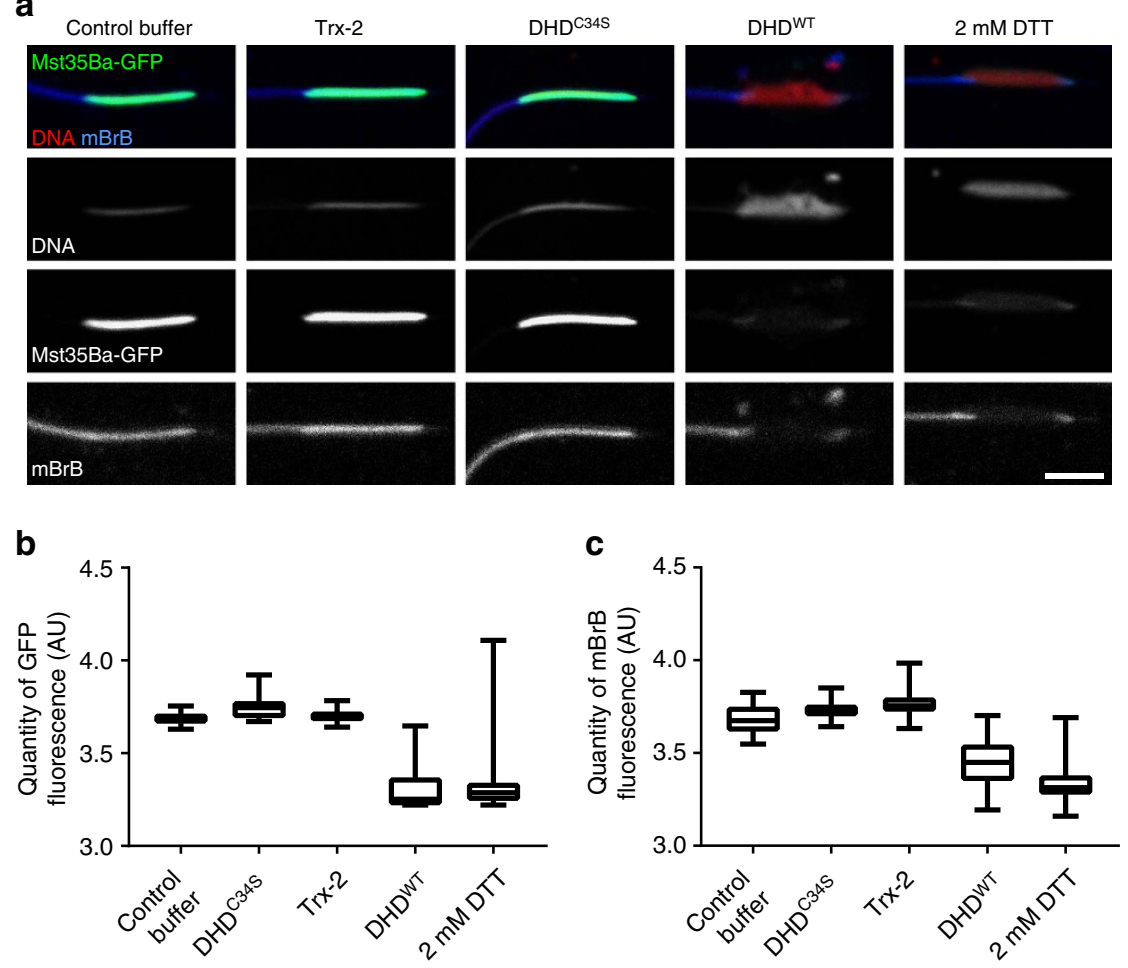

Figure 7 | DHD protein is sufficient to decondense sperm nuclei in vitro. (a) Confocal images of Mst35Ba-GFP sperm nuclei incubated with recombinant thioredoxins. The DHDWT recombinant thioredoxin induces nuclear decondensation and Mst35Ba-GFP removal in a way similar to DTT treatment. The sperm nuclei incubated with DHDC34S or Trx-2 recombinant proteins appear similar to sperm nuclei incubated with control buffer. Green: Mst35Ba-GFP, Blue: $\mathrm{mBrB}$, Red: DNA. (b,c) Quantification of native GFP signal (b) or $\mathrm{mBrB}$ fluorescence (c) from sperm nuclei incubated with the recombinant thioredoxins. Error bars indicate SD calculated for 30 analysed nuclei for each experiment. Scale bar, $5 \mu \mathrm{m}$.

prevent further access of the thioredoxin to the compact sperm nucleus. We thus propose that, following sperm penetration through the egg micropyle and sperm plasma membrane breakdown, the sperm nucleus is exposed to the egg cytoplasm that contains massive amounts of DHD thioredoxin. The reduction of disulfide crosslinks by DHD induces sperm nuclear decompaction and allows the subsequent extraction of SNBPs from sperm chromatin, a process that likely involves the ISWI chromatin remodeler ${ }^{33}$. Finally, the removal of SNBP leaves DNA accessible to the HIRA complex for genome-wide nucleosome assembly with maternally supplied histones ${ }^{34-36}$.

In mammals, the tripeptide antioxidant glutathione is abundant in oocytes and plays an important role in sperm nuclear decondensation ${ }^{44,47,48}$. Inhibition of glutathione synthesis by buthionine sulfoximine during oocyte maturation indeed efficiently blocks sperm nuclear decondensation and this effect can be reversed by DTT (refs 47,48). Whether glutathione directly reduces SNBP disulfides in vivo or instead activates a dedicated disulfide reductase is still unresolved ${ }^{49}$. In most species, glutathione disulfide (GSSG) is reduced into glutathione (GSH) by the highly conserved glutathione reductase enzyme. Drosophila is unusual in lacking glutathione reductase and instead relies on the thioredoxin system for GSSG reduction ${ }^{50}$. Interestingly, biochemical characterization of Drosophila thioredoxins have established that DHD and Trx-2 are equally efficient for GSSG reduction in vitro ${ }^{50,51}$, opening the possibility that DHD could indirectly influence sperm nuclear decondensation by controlling the level of GSH in eggs. However, our demonstration that recombinant DHD protein is sufficient for sperm nuclear decondensation and SNBP removal in our in vitro assay argues against this hypothesis. Still, we have clearly documented that sperm chromatin remodelling is not completely blocked in dhd eggs but instead occurs very slowly. This slow replacement of SNBPs with histones suggests that mutant eggs still have residual reducing power for the cleavage of SNBP disulfide bonds, an effect that could likely involve glutathione itself.

Our study also revealed that the migration of the female pronucleus to the centre of the egg fails in a majority of $d h d$ eggs, thus preventing the initiation of embryo development. We found that the release of paternal centrioles from the base of the sperm nucleus and the subsequent elaboration of the sperm aster were indeed affected in mutant eggs. As a matter of fact, depletion of glutathione in bovine oocytes not only blocks sperm nuclear decondensation but also prevents sperm aster growth and pronuclear migration ${ }^{48}$. The authors indeed observed that the disassembly of the sperm tail and connecting piece, as well as the release of the proximal centriole were frequently affected in treated oocytes, suggesting that the role of glutathione at fertilization is not restricted to sperm chromatin decompaction. Further investigation will be required to determine whether DHD similarly targets additional sperm structures.

In conclusion, we propose that the rapid unlocking of sperm chromatin at fertilization in Drosophila is critically dependent on the redox activity of the egg specific DHD thioredoxin. dhd is apparently the most recent thioredoxin gene in Drosophila and likely originated after the duplication of the ancestral $\operatorname{Tr} x-2$ gene $^{18}$. It is thus tempting to propose that the emergence of this highly specialized protein could reflect the adaptation of the oocyte to the rapid evolution of sperm chromatin architecture. Future work will aim at understanding how the structural features of DHD underlie its crucial specialization at the onset of embryo development. 


\section{Methods}

Fly stocks. Flies were raised at $25^{\circ} \mathrm{C}$ on standard cornmeal-agar-yeast medium. The $w^{1118}$ strain was used as a wild-type control. The $D f(1) J 5 / F M 7 c$ and $D f(1) J 5 / D f(1) J 5 ; C y O, P\left[\right.$ dhd Xhol/XhoI,$\left.w^{+}\right] /+$stocks $^{19}$ were obtained from $\mathrm{H}$ Salz. The boundaries of the genomic fragment carried by $P\left[d h d^{X h o I / X h o l}, w^{+}\right]$ transgene (see Fig. 1a) were verified by PCR and DNA sequencing. We additionally used the following stocks: $P[s n k y-G F P] ; s n k y^{1}$ (ref. 30), P[EGFP-cid] (ref. 52), $\Delta M s t 35 B$ (ref. 39), $P[M s t 35 B a-E G F P]$ (ref. 37), $P\left[\right.$ Mst77F-EGFP] (ref. 31), snky ${ }^{1}$ (ref. 27) and $P[D j-G F P]$ (ref. 53). Additional stocks were obtained from the Bloomington Drosophila stock centre: $D f(1) J C 70$ (\#944) is a deficiency of the cytogenetic region 4C11-5A4 which does not complement the female sterility associated with $d h d^{5}$ (Table 1); Df(3L)BSC12 (\#6457) is a deficiency that uncovers the snky locus. The $p W 8$-attB $\left[d h d^{W T}\right]$ and $p W 8$-attB $\left[d h d^{C 34 S}\right]$ transgenic lines were generated by PhiC31 mediated insertion ${ }^{54}$ into the $P B a c\left\{y^{+}\right.$-attP3B\}VK00031 platform (62E1).

Fertility tests. Virgin females were aged for 3 days at $25^{\circ} \mathrm{C}$ in the presence of males and were then allowed to lay eggs on standard medium for $24 \mathrm{~h}$. Embryos were counted and then let to develop at least $36 \mathrm{~h}$ at $25^{\circ} \mathrm{C}$. Unhatched embryos were counted to determine hatching rates.

Plasmid constructs. $p W 8$-attB[dhd $\left.d^{W T}\right]$. A 4303 bp genomic fragment $\left(d h d^{W T}\right.$ ) containing the $d h d$ coding sequence, upstream (1932 bp) and downstream (2047 bp) regions, was amplified from wild-type genomic DNA using the following primers: $5^{\prime}$-GCCGGTACCCCCATATCCCTCCCATATCC-3' and 5'-GCCGGAT CCGCTAATGGAATCGCAATCGT- $3^{\prime}$ and cloned into the $p W 8$-attB plasmid ${ }^{55}$. $p W 8$-attB $\left[d h d^{\mathrm{C} 34 S}\right]$. The $d h d^{\mathrm{C} 34 \mathrm{~S}}$ fragment was obtained by in vitro mutagenesis of $d h d^{W T}$ plasmid using the QuickChange II XL Site-Directed Mutagenesis Kit (Agilent Technologies) with the following primers: 5'-CATGGTGTGGTCCCAGC AAGGAAATGGAGAGC- $3^{\prime}$ and $5^{\prime}$-GCTCTCCATTTCCTTGCTGGGACCACA CCATG- $3^{\prime}$ and cloned into $p W 8$-attB.

$p E T 15 B\left(d h d^{W T}\right)$ and $p E T 15 b\left(d h d^{C 34 S}\right)$. The $d h d^{W T}$ and $d h d^{C 34 S}$ fragments were amplified from the $p W 8-a t t B\left(d h d^{W T}\right)$ and $p W 8$-attB $\left(d h d^{C 34 S}\right)$ plasmids, respectively, with the following primers $5^{\prime}$-TAATTCCATATGATGGCATC CGTACGCACCATG- $3^{\prime}$ and

$5^{\prime}$-TAATTCGGATCCTTACGCCTTCACCAGCTTGGC- $3^{\prime}$ and cloned into pET15b.

$p E T 15 b(\operatorname{Tr} x-2)$. The $T r x-2$ coding sequence was synthetized, amplified with the following primers $5^{\prime}$-TAATTCCATATGATGGTGTACCAGGTGAAAGAT- $3^{\prime}$ and $5^{\prime}$-TAATTCGGATCCTTAGATATTGGCCTTGATGACA- $3^{\prime}$ and cloned into $p E T 15 b$.

DHD antibody production. A rabbit polyclonal anti-DHD antiserum was raised against a mixture of three synthetic peptides (DHD-1: MASVRTMNDYHKRIEA ADC, DHD-2: CKEMESTVKSLARKYSSKA, DHD-3: CAGADEHKLTNMM AKLVKA). DHD antibody was purified by affinity chromatography.

Embryos preparation and imaging. Early $(0-30 \mathrm{~min})$ and late $(0-120 \mathrm{~min})$ embryos were collected on agar plates. Embryos were dechorionated in bleach, fixed in a 1:1 heptane:methanol mixture and stored at $-20^{\circ} \mathrm{C}$. For immunostaining experiments, embryos were washed three times $(10 \mathrm{~min}$ each) in PBS, $0.1 \%$ Triton X100. Embryos were incubated with primary antibodies in the same buffer on a wheel overnight at $4{ }^{\circ} \mathrm{C}$ and washed three times (20 min each) in PBS, $0.1 \%$ Triton X100. Incubation with secondary antibodies was performed identically. For propidium iodide staining, embryos were incubated for $1 \mathrm{~h}$ in a $2 \mathrm{mg}$ per $\mathrm{ml}$ RNAse A solution at $37^{\circ} \mathrm{C}$. Embryos were finally mounted in mounting medium (Dako) containing propidium iodide (Sigma) or DAPI.

Primary antibodies used were: mouse monoclonal anti-GFP (Roche \#118144600001; 1:200), rabbit polyclonal anti-H3K27me2 (Millipore \#07-452; 1:500), mouse monoclonal anti-histones (Millipore \#MABE71; 1:1,000), mouse monoclonal anti- $\gamma$-Tubulin (Sigma \#T6557; 1:500), rat monoclonal anti- $\alpha$-Tubulin (Abcam \#ab6160; 1:50), rabbit polyclonal anti-DHD $(1: 1,000)$. Secondary antibodies were used at a 1:1,000 dilution and included goat anti-mouse and goat anti-rabbit antibodies conjugated to DyLight (Thermoscientific), as well as goat anti-rat and goat anti-mouse conjugated to AlexaFluor (Jackson

ImmunoResearch). Images were acquired on a LSM 510 META (Carl Zeiss) or a TCS SP5X (Leica) confocal microscope and the images were processed with ImageJ and Inkscape softwares.

Expression and preparation of recombinant thioredoxins. E. coli BL21 cells (Agilent Technoligies \#230134) were transformed with the different pET15b constructs and their expression were induced using $1 \mathrm{mM}$ IPTG (isopropyl $\beta$-D-thiogalactoside) for $2 \mathrm{~h}$ at $37^{\circ} \mathrm{C}$. Cell lysis and protein purification were performed with the Protino Ni-NTA agarose kit (Macherey-Nagel) according to the manufacturer instructions. Before incubation with sperm nuclei, the recombinant thioredoxins were reduced for $3 \mathrm{~h}$ at room temperature with $10 \mathrm{mM}$ DTT and desalted with PD-10 Desalting Column containing Sephadex G25 Medium (GE Healthcare) to remove excess DTT. Protein elution was performed using $0.1 \mathrm{M}$
Potassium Phosphate Buffer pH 7.0, 1 mM EDTA. The control buffer used in decondensation assays is $0.1 \mathrm{M}$ Potassium Phosphate Buffer $\mathrm{pH}$ 7.0, $1 \mathrm{mM}$ EDTA; $10 \mathrm{mM}$ DTT eluted from PD10 Desalting column.

Preparation and imaging of sperm nuclei treated with DTT. Virgin males were collected five days before dissection. Seminal vesicles were dissected in 1X PBS; 1\% Triton X100 and torn open with tweezers to release spermatozoa on polylysine slides. Slides were first incubated at room temperature in IX PBS; 1\% Triton X100 for $30 \mathrm{~min}$, air dried and then incubated in $1 \mathrm{X}$ PBS; $2 \mathrm{mM}$ DTT for $0,1,5,10,15$ or $30 \mathrm{~min}$ at room temperature. Slides were quickly washed in $1 \mathrm{X}$ PBS, incubated for $15 \mathrm{~min}$ at room temperature in $1 \mathrm{mM} \mathrm{mBrB}$ (Millipore \#596105), quickly washed and mounted in mounting medium (Dako) containing propidium iodide (Sigma)

Decondensation of sperm nuclei with recombinant thioredoxins. Seminal vesicles from virgin males were dissected as described above. Slides were incubated at room temperature in 1X PBS 1\%, Triton X100 for $30 \mathrm{~min}$, air dried and then incubated 3 times for $30 \mathrm{~min}$ in Potassium Phosphate Buffer containing the desalted thioredoxins. Slides were quickly washed in 1X PBS. Before quantification, slides were incubated for $15 \mathrm{~min}$ at room temperature in $1 \mathrm{mM} \mathrm{mBrB}$ (Millipore \#596105), quickly washed and mounted in mounting medium (Dako) containing propidium iodide (Sigma).

Imaging and fluorescence quantification of sperm nuclei. Sperm nuclei were imaged on a TCS SP5 X confocal microscope (Leica) or on a LSM 800 confocal microscope (Carl Zeiss). The images were analysed using the ImageJ 1.48 software to measure the total quantity of fluorescence. Each nucleus was counted as an individual object in a defined area and the background signal outside the nucleus was used to determine the fluorescence baseline. Within the selected area, the number of pixels for each value of fluorescence (ranging from the baseline to 255) was counted. Finally, the total quantity of fluorescence was calculated with the following formula:

$\left[\sum_{i=x}^{255} n(i) \times i\right] / 10,000$ in which $i$ is the fluorescence value, $x$ is the baseline fluorescence and $n(i)$ is the number of pixels for the fluorescence value $i$.

$\mathrm{PI}$ and GFP signals were quantified in the same experiment and $\mathrm{mBrB}$ signal in an independent one. Each experiment was performed twice and gave similar results. Statistical tests (Mann Whitney) were performed using Prism (Graphpad) software.

Western Blotting. Ovaries or testes from 30 individuals were collected and homogenized in lysis buffer $(20 \mathrm{mM}$ Hepes $\mathrm{pH} 7.9,100 \mathrm{mM} \mathrm{KCl}, 0.1 \mathrm{mM}$ EDTA, $0.1 \mathrm{mM}$ EGTA, 5\% Glycerol, $0.05 \%$ NP40 and protease inhibitors (Roche)). The protein extracts were cleared by centrifugation and stored at $-80^{\circ} \mathrm{C}$.

Eggs were collected every $30 \mathrm{~min}$ and incubated for 0,15 or $30 \mathrm{~min}$ at $25^{\circ} \mathrm{C}$. They were then dechorionated in bleach and quickly frozen in liquid nitrogen. Protein extracts were prepared from $\sim 10 \mu \mathrm{L}$ of embryos, as described for gonads.

Electrophoresis was carried out on 5-20\% gradient SDS polyacrylamide gel and Western blotting was performed using the ECL prime Western blotting detection reagent and following the manufacturer's instructions (GE Healthcare). Uncropped images are in Supplementary Fig. 6.

The antibodies were: mouse monoclonal anti- $\alpha$-Tubulin (Sigma \#T9026, 1:500), rabbit polyclonal anti-DHD (1:1,000), rabbit polyclonal anti-Trx- $2^{51}(1: 1,000$; a gift from Katja Becker), HRP-conjugated goat anti-mouse (Biorad \#170-5047; 1:50,000) and peroxidase-conjugated goat anti-rabbit (Thermoscientific \#32460; $1: 20,000)$.

RNA probe synthesis. A 436 bp $d h d$ sequence was amplified using primers containing the T3 or T7 promoter sequences (T3-dhd-f: $5^{\prime}$-AATTAACCCTCAC TAAAGGGCGACGACAAGCTAATCGTGC-3', $77-d h d-r$ : $5^{\prime}$-TAATACGACTC ACTATAGGGTACCCACATCTCGCGCTTAC- $3^{\prime}$ ). A DIG labelled RNA antisense probe was directly transcribed from the purified PCR amplicon with T7 RNA polymerase and a labelling mix (Roche).

RNA in situ hybridization. Ten pairs of ovaries from 1 to 5 day-old virgin females were dissected in $1 \mathrm{X}$ PBS; $0.2 \%$ Tween 20 (PBST), fixed for 30 min in a 1:1 mixture of 3.7\% formaldehyde-PBST:heptane. After PBST washings, the tissues were postfixed with $3.7 \%$ formaldehyde-PBST for $10 \mathrm{~min}$, then washed in PBST, and sequentially incubated in 1:1 PBST/hybridization buffer (HB) (50\% formamide, $5 \mathrm{X}$ SSC, $0.1 \%$ Tween 20 , pH 6.5) for $10 \mathrm{~min}$, in $100 \% \mathrm{HB}$ for $10 \mathrm{~min}$, and finally in $\mathrm{HB}$ containing $100 \mu \mathrm{g} \mathrm{ml}^{-1}$ tRNA and $50 \mu \mathrm{g} \mathrm{ml}^{-1}$ heparin, for $1 \mathrm{~h}$ at $65^{\circ} \mathrm{C}$. Then, the probe was added before overnight incubation at $65^{\circ} \mathrm{C}$. The ovaries were then washed twice with $\mathrm{HB}$ at $65^{\circ} \mathrm{C}$, and once with 1:1 PBST:HB at $65^{\circ} \mathrm{C}$. DIG-labelled RNA probes detection was performed using anti-DIG AP antibody (Roche \#11093274910; 1:100) and NBT/BCIP colorimetric detection was carried out according to manufacturer instructions (Roche). Ovaries were mounted in mounting medium (Dako) and were imaged using a Z1 microscope (Carl Zeiss).

Data Availability. All relevant data are available from the authors on request. 


\section{References}

1. Miller, D., Brinkworth, M. \& Iles, D. Paternal DNA packaging in spermatozoa: more than the sum of its parts? DNA, histones, protamines and epigenetics. Reproduction 139, 287-301 (2010)

2. Rathke, C., Baarends, W. M., Awe, S. \& Renkawitz-Pohl, R. Chromatin dynamics during spermiogenesis. BBA-Gene Regul. Mech. 1839, 155-168 (2014).

3. Lewis, J. D., Song, Y., Jong, M. E., Bagha, S. M. \& Ausió, J. A walk though vertebrate and invertebrate protamines. Chromosoma 111, 473-482 (2003).

4. Gaucher, J. et al. From meiosis to postmeiotic events: the secrets of histone disappearance. FEBS J. 277, 599-604 (2010).

5. Eirin-López, J. M. \& Ausió, J. Origin and evolution of chromosomal sperm proteins. Bioessays 31, 1062-1070 (2009).

6. Oliva, R. Protamines and male infertility. Hum. Reprod. Update 12, 417-435 (2006).

7. Balhorn, R. The protamine family of sperm nuclear proteins. Genome Biol. 8, 227 (2007).

8. Balhorn, R. A model for the structure of chromatin in mammalian sperm. J. Cell Biol. 93, 298-305 (1982).

9. Ward, W. S. Function of sperm chromatin structural elements in fertilization and development. Mol. Hum. Reprod. 16, 30-36 (2010).

10. Bedford, J. M. \& Calvin, H. I. Changes in -S-S- linked structures of the sperm tail during epididymal maturation, with comparative observations in sub-mammalian species. J. Exp. Zool. 187, 181-204 (1974).

11. Vilfan, I. D., Conwell, C. C. \& Hud, N. V. Formation of native-like mammalian sperm cell chromatin with folded bull protamine. J. Biol. Chem. 279, 20088-20095 (2004).

12. Bjorndahl, L. \& Kvist, U. Human sperm chromatin stabilization: a proposed model including zinc bridges. Mol. Hum. Reprod. 16, 23-29 (2010).

13. Collet, J.-F. \& Messens, J. Structure, function, and mechanism of thioredoxin proteins. Antioxid. Redox Signal 13, 1205-1216 (2010).

14. Berndt, C., Schwenn, J.-D. \& Lillig, C. H. The specificity of thioredoxins and glutaredoxins is determined by electrostatic and geometric complementarity. Chem. Sci. 6, 7049-7058 (2015).

15. Svensson, M. \& Larsson, J. Thioredoxin-2 affects lifespan and oxidative stress in Drosophila. Hereditas 144, 25-32 (2007).

16. Tsuda, M. et al. Loss of Trx-2 enhances oxidative stress-dependent phenotypes in Drosophila. FEBS Lett. 584, 3398-3401 (2010).

17. Svensson, M. J., Chen, J. D., Pirrotta, V. \& Larsson, J. The ThioredoxinT and deadhead gene pair encode testis- and ovary-specific thioredoxins in Drosophila melanogaster. Chromosoma 112, 133-143 (2003).

18. Svensson, M. J., Stenberg, P. \& Larsson, J. Organization and regulation of sex-specific thioredoxin encoding genes in the genus Drosophila. Dev. Genes Evol. 217, 639-650 (2007)

19. Salz, H. K. et al. The Drosophila maternal effect locus deadhead encodes a thioredoxin homolog required for female meiosis and early embryonic development. Genetics 136, 1075-1086 (1994).

20. Pellicena-Palle, A., Stitzinger, S. M. \& Salz, H. K. The function of the Drosophila thioredoxin homologue encoded by the deadhead gene is redox-dependent and blocks the initiation of development but not DNA synthesis. Mech. Dev. 62, 61-65 (1997).

21. Fuyama, Y. Gynogenesis in Drosophila melanogaster. Jpn. J. Genet. 59, 91-96 (1984).

22. Loppin, B., Docquier, M., Bonneton, F. \& Couble, P. The maternal effect mutation sésame affects the formation of the male pronucleus in Drosophila melanogaster. Dev. Biol. 222, 392-404 (2000).

23. Loppin, B., Berger, F. \& Couble, P. Paternal chromosome incorporation into the zygote nucleus is controlled by maternal haploid. in Drosophila Dev. Biol. 231, 383-396 (2001).

24. Karr, T. L. Paternal investment and intracellular sperm-egg interactions during and following fertilization in Drosophila. Curr. Top. Dev. Biol. 34, 89-115 (1996).

25. Loppin, B., Dubruille, R. \& Horard, B. The intimate genetics of Drosophila fertilization. Open Biol 5, 150076 (2015).

26. Foe, V., Odell, G. M. \& Edgar, B. A. In The Development of Drosophila melanogaster. (eds Bate, M. \& Martinez Arias, A.) 149-300 (Cold Spring Harbor Laboratory Press, 1993).

27. Fitch, K. R. \& Wakimoto, B. T. The paternal effect gene $m s(3)$ sneaky is required for sperm activation and the initiation of embryogenesis in Drosophila melanogaster. Dev. Biol. 197, 270-282 (1998).

28. Ohsako, T., Hirai, K. \& Yamamoto, M.-T. The Drosophila misfire gene has an essential role in sperm activation during fertilization. Genes Genet. Syst. 78, 253-266 (2003)

29. Ohsako, T. \& Yamamoto, M.-T. Sperm of the wasted mutant are wasted when females utilize the stored sperm in Drosophila melanogaster. Genes Genet. Syst. 86, 97-108 (2011)

30. Wilson, K. L., Fitch, K. R., Bafus, B. T. \& Wakimoto, B. T. Sperm plasma membrane breakdown during Drosophila fertilization requires Sneaky, an acrosomal membrane protein. Development 133, 4871-4879 (2006).
31. Jayaramaiah Raja, S. \& Renkawitz-Pohl, R. Replacement by Drosophila melanogaster protamines and Mst77F of histones during chromatin condensation in late spermatids and role of sesame in the removal of these proteins from the male pronucleus. Mol Cell Biol 25, 6165-6177 (2005). (Erratum in: Mol. Cell. Biol. 26, 3682 2006).

32. Eren-Ghiani, Z., Rathke, C., Theofel, I. \& Renkawitz-Pohl, R. Prtl99C acts together with protamines and safeguards male fertility in Drosophila. Cell Reports 13, 2327-2335 (2015).

33. Doyen, C. M. et al. A testis-specific chaperone and the chromatin remodeler ISWI mediate repackaging of the paternal genome. Cell Reports 13, 1310-1318 (2015).

34. Loppin, B. et al. The histone H3.3 chaperone HIRA is essential for chromatin assembly in the male pronucleus. Nature 437, 1386-1390 (2005).

35. Bonnefoy, E., Orsi, G. A., Couble, P. \& Loppin, B. The essential role of Drosophila HIRA for de novo assembly of paternal chromatin at fertilization. PLoS Genet. 3, e182 (2007)

36. Orsi, G. A. et al. Drosophila Yemanuclein and HIRA cooperate for de novo assembly of H3.3-containing nucleosomes in the male pronucleus. PLoS Genet 9, e1003285 (2013).

37. Manier, M. K. et al. Resolving mechanisms of competitive fertilization success in Drosophila melanogaster. Science 328, 354-357 (2010).

38. Rathke, C. et al. Distinct functions of Mst77F and protamines in nuclear shaping and chromatin condensation during Drosophila spermiogenesis. Eur J. Cell Biol. 89, 326-338 (2010).

39. Tirmarche, S. et al. Drosophila Protamine-Like Mst35Ba and Mst35Bb are required for proper sperm nuclear morphology but are dispensable for male fertility. G3 (Bethesda) 4, 2241-2245 (2014).

40. Alvi, Z. A., Chu, T.-C., Schawaroch, V. \& Klaus, A. V. Protamine-like proteins in 12 sequenced species of Drosophila. Protein Pept. Lett. 20, 17-35 (2013).

41. Kanippayoor, R. L., Alpern, J. H. M. \& Moehring, A. J. Protamines and spermatogenesis in Drosophila and Homo sapiens. Spermatogenesis 3, e24376 (2014).

42. Oliva, R. \& Dixon, G. H. Vertebrate protamine gene evolution I. Sequence alignments and gene structure. J. Mol. Evol. 30, 333-346 (1990).

43. Gimenez-Bonafé, P. et al. Chromatin condensation, cysteine-rich protamine, and establishment of disulphide interprotamine bonds during spermiogenesis of Eledone cirrhosa (Cephalopoda). Eur J. Cell Biol. 81, 341-349 (2002).

44. Zirkin, B. R., Perreault, S. D. \& Naish, S. J. in The Molecular Biology of Fertilization (eds, Schatten. H \& Schatten, G.) 91-114 (Academic Press, New York, 1989).

45. Kost, N. et al. Multimerization of Drosophila sperm protein Mst77F causes a unique condensed chromatin structure. Nucleic Acids Res. 43, 3033-3045 (2015).

46. Konev, A. Y. et al. CHD1 motor protein is required for deposition of histone variant H3.3 into chromatin in vivo. Science 317, 1087-1090 (2007).

47. Perreault, S. D., Barbee, R. R. \& Slott, V. L. Importance of glutathione in the acquisition and maintenance of sperm nuclear decondensing activity in maturing hamster oocytes. Dev. Biol. 125, 181-186 (1988).

48. Sutovsky, P. \& Schatten, G. Depletion of glutathione during bovine oocyte maturation reversibly blocks the decondensation of the male pronucleus and pronuclear apposition during fertilization. Biol. Reprod. 56, 1503-1512 (1997).

49. Li, J. et al. Identification and characterization of an oocyte factor required for sperm decondensation in pig. Reproduction 148, 367-375 (2014).

50. Kanzok, S. M. Substitution of the thioredoxin system for glutathione reductase in Drosophila melanogaster. Science 291, 643-646 (2001).

51. Bauer, H., Kanzok, S. M. \& Heiner Schirmer, R. Thioredoxin-2 but not thioredoxin-1 is a substrate of thioredoxin peroxidase-1 from Drosophila melanogaster. J. Biol. Chem. 277, 17457-17463 (2002).

52. Schuh, M., Lehner, C. F. \& Heidmann, S. Incorporation of Drosophila CID/CENP-A and CENP-C into centromeres during early embryonic anaphase. Curr. Biol. 17, 237-243 (2007).

53. Santel, A., Winhauer, T., Blümer, N. \& Renkawitz-Pohl, R. The Drosophila don juan (dj) gene encodes a novel sperm specific protein component characterized by an unusual domain of a repetitive amino acid motif. Mech. Dev. 64, 19-30 (1997).

54. Bischof, J., Maeda, R. K., Hediger, M., Karch, F. \& Basler, K. An optimized transgenesis system for Drosophila using germ-line-specific phiC31 integrases. Proc. Natl Acad. Sci. USA 104, 3312-3317 (2007).

55. Dubruille, R. et al. Specialization of a Drosophila capping protein essential for the protection of sperm telomeres. Curr. Biol. 20, 2090-2099 (2010).

\section{Acknowledgements}

We are grateful to Helen Salz for her generous gift of the dhd mutant stock. Additional stocks were kindly provided by Christina Rathke, John Belote, Barbara Wakimoto and the Bloomington Drosophila Stock Center. We also thank Katja Becker for the gift of the anti-Trx-2 antibody and Gérard Benoit for his critical reading of the manuscript. Confocal microscopy was performed at the Centre Technologique des Microstructures 
and the Centre d'Imagerie Quantitative Lyon Est (UCBL Lyon1). This work was supported by a grant from the Fondation pour la Recherche Médicale (DEP20131128256) and a grant from the Agence Nationale de la Recherche (ZygoPat-ANR-12-BSV6-0014).

\section{Author contributions}

S.T. performed most of the experiments. S.K. performed in situ hybridizations and bioinformatic analyses. R.D. designed and cloned some of the constructs. S.T., B.H. and B.L. designed the experiments and analysed the data. B.L. wrote the paper. All authors discussed the results and commented on the manuscript.

\section{Additional information}

Supplementary Information accompanies this paper at http://www.nature.com/ naturecommunications

Competing financial interests: The authors declare no competing financial interests.
Reprints and permission information is available online at http://npg.nature.com/ reprintsandpermissions/

How to cite this article: Tirmarche, S. et al. Unlocking sperm chromatin at fertilization requires a dedicated egg thioredoxin in Drosophila. Nat. Commun. 7, 13539 doi: $10.1038 /$ ncomms13539 (2016).

Publisher's note: Springer Nature remains neutral with regard to jurisdictional claims in published maps and institutional affiliations.

(c) (i) This work is licensed under a Creative Commons Attribution 4.0 International License. The images or other third party material in this article are included in the article's Creative Commons license, unless indicated otherwise in the credit line; if the material is not included under the Creative Commons license, users will need to obtain permission from the license holder to reproduce the material. To view a copy of this license, visit http://creativecommons.org/licenses/by/4.0/

C) The Author(s) 2016 